\section{The Air Defense Game: A microcomputer program for research in human performance}

\author{
FRANK L. GREITZER, RAMON L. HERSHMAN, \\ and RICHARD T. KELLY \\ Navy Personnel Research and Development Center, \\ San Diego, California 92152
}

The study of human performance in complex tasks is often impeded by the tradeoff between external validity and proper control of environmental and task variables. We encountered this problem in investigations of humancomputer interactions in the U.S. Navy command and control environment.

We sought a laboratory research task that required sustained attention and timely decisions by the subject, operated in real-time, and appropriately changed the environment in response to the subject's decision actions. The task had to be reasonably complex, yet not too difficult to perform. It also had to be highly motivating and provide performance measures sufficient for detailed quantitative analysis. The task also had to represent significant features of a Navy tactical problem.

The selected problem embedded elements of an air defense operation in an interactive game that was controlled by a Tektronix 4051 microcomputer (Kelly, Greitzer, \& Hershman, Note 1). The major features and applications of the Air Defense Game are described here.

\section{Game Scenario}

The game simulates a radar screen on which hostile air targets approach the player's ship at one of three speeds and the player defends by launching "missiles." The entire scenario unfolds on the computer's graphic CRT. A sample snapshot of the game in progress appears in Figure 1. The "+" at the center of the display is the ship. Its detection range (outer circle) exceeds its weapon range (inner circle), so that the player must decide, on the basis of target speed, when to launch each missile. The goal is to destroy all targets at maximum range, but weapons that are launched too soon fall short (splash) and are ineffective. No more than one missile is permitted in-flight on a given track at a time; duplicate, or in-flight, launches result in a penalty.

Targets are identified by a two-digit track number (TN) and enter the display as dots ("blips") at the outer circle. Each target heads directly toward the ship, and its position is updated at 11.7 -sec intervals by another blip that corresponds to the next sweep of the simulated radar. The player launches a missile at a target by entering its TN via coded function keys on the Tektronix 4051

The opinions contained herein are those of the authors and are not to be construed as official or reflecting the views of the Navy Department.

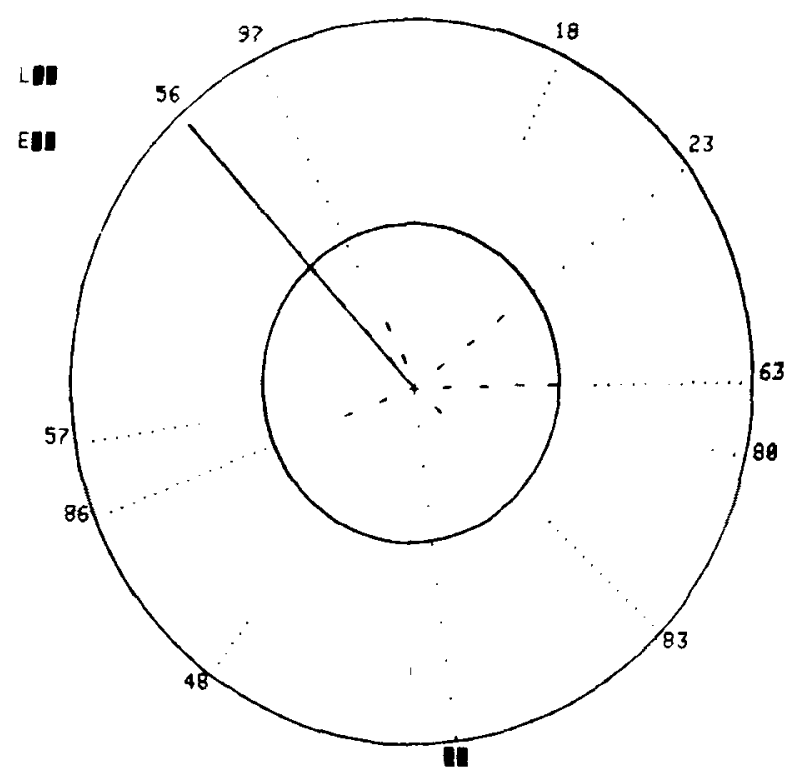

Figure 1. CRT display of a single update in the Air Defense Game. The distance beween target blips indicates relative speed: TN 23 is a fast target, TN 80 is medium, and TN 57 is slow. The dashes are the player's missiles. TNs $23,83,86$, and 97 are engaged. TN 56 has been destroyed, and the target at 6 o'clock has scored a hit. A splash has occurred on TN 63. The radius of the outer circle is $13.5 \mathrm{~cm}$.

keyboard. Feedback is flashed at the left of the display and indicates either a good launch or an error. Errors occur if the keyed TN does not exist or if a missile is already in flight at the entered TN. A dash (-) represents the player's missile. It has the same speed as a fast target, and its position is also updated during each radar sweep. Visual and auditory feedback are given each time a missile destroys a target (a kill) and when a target impacts the ship (a hit). A full play of the game constitutes an engagement, which ends when all targets have either been killed or have hit the ship. The display is a storage-tube device, so that all video remains on the screen for the duration of an engagement.

\section{Task Variables}

The major variables affecting the game are the total number of targets, target speed, and the tempo of operation.

Number of targets. Selection from a menu provides $6,18,36,54$, or 72 total targets in an engagement. Although spatial constraints allow a maximum of 90 targets ( 4 deg between neighboring tracks), limitations in computer memory forced the 72-target ceiling.

Target speed. Targets advance at one of three constant speeds (fast, medium, or slow), and equal numbers of each are always assigned. Speed clearly determines the optimal time to fire, that is, the update at which a launch will achieve the maximum kill range. 
Tempo of operation. This important variable controls the rate at which targets appear. Three options are selectable by menu. In the low-tempo condition, entry times of the individual targets are relatively spread out and the processing load is light. As the tempo is raised to intermediate and high, the times of entry are increasingly crowded together and the information processing demands increase. While targets enter throughout the engagement, the game parameters create a peak demand for decision and action in the middle of an engagement. The effect of the tempo variable is to vary the magnitude of this peak.

\section{Performance Analysis}

The computer records the player's actions and displays selected feedback at the end of each engagement. This summary includes the average range for kills, the total number of kills and hits, the number of splashes and in-flight launches, and a skill rating $(R)$ that reflects overall proficiency. $R$ is defined by: $R=100$ (average kill range/20) - 12(number of hits) - 2(number of inflights). If performance is perfect, all targets will be destroyed at the maximum range of 20 miles and there will be no hits or in-flight launches; then, $R=100$. More fine-grained measures can be obtained with a separate off-line program that analyzes the data stored on magnetic tape. Excerpts from this analysis are summarized below; see Kelly et al. (Note 1) for more details.

For each target, there is an optimal update on which to fire that depends only on the target's speed and time of entry. This permits a specification of optimal performance for each track and thus for each engagement. As one performance measure, we compare the player's actions to the optimal tactics by taking the difference between observed launch time (L) and optimal launch time $\left(\mathrm{L}^{*}\right)$ to yield the lag $=\left(\mathrm{L}-\mathrm{L}^{*}\right)$. The ideal player will have zero lags for all targets. However, real players have limited processing resources and cannot respond to all tracks as they first become eligible. For experienced players, the mean lag varies inversely with target speed. Presumably, this follows from the priorities imposed by the scoring rules. For instance, slow-speed targets move the shortest distance between updates, so that smaller penalties are incurred by firing at fast and medium ones instead.

Task load is not constant, but rather, it peaks in the middle of the engagement. To explore the response to this time-varying information load, the analysis program also captures a dynamic profile of the player's efficiency as the game unfolds in time. Players invariably fall further behind, relative to optimal performance, during the middle, higher paced portion of the engagement. Efficiency then recovers as the processing burden decreases toward the game's end.

\section{Modeling}

The game provides fertile ground for modeling vari- ous aspects of performance. For example, we sought a unidimensional measure of task difficulty that combined the major task variables: total number of targets and the tempo of operation. We identified the difficulty at any update with the presumed processing load for the optimal player. This load was based on a simple count of the number of targets in close proximity (suitably defined) to their firing range. The sum, over updates, of these counts above a given threshold (the limit of supposed processing capacity) then provided a measure of difficulty for an engagement. The derived difficulty measure is linear with the number of targets; the slope increases with the tempo of operation. Finally, the performance of real players as evidenced by their skill ratings ( $R$ ) and lags is a strict linear function of the modeled difficulty metric.

\section{Notes on Implementation}

The total time for an engagement depends largely on the selected task variables and changes only slightly with the player's proficiency and the built-in randomization of the targets' entry times. The range is from approximately $7.1 \mathrm{~min}$ for the shortest 18-target scenario to $24.8 \mathrm{~min}$ for the longest 72 -target scenario. The task loadings of greatest research interest are those at high tempo with $18,36,54$, or 72 targets; the approximate game durations are $4.2,6.0,8.0$, and $9.9 \mathrm{~min}$, respectively.

With Navy enlisted men as subjects, we have found that $3 \mathrm{~h}$ of practice produces proficient and stable performance at the moderately difficult, high-tempo, 36-target condition. Total acquisition time is virtually insensitive to training sequence. The six-target practice task is recommended for initial training to a criterion score of $\mathrm{R}=90$; any progression through the other conditions is then adequate.

This game has been used to study the effects of various task loads and stress, including dual-task performance, prolonged periods of wakefulness, and physical activity. Further refinement of the simulation is in progress for future applications. Unlike most tasks in the laboratory, the air defense simulation is fun to play. It generates high motivation for subjects and sustains participants' interest for $34 \mathrm{~h}$ at a sitting.

\section{Programming Language and Requirements}

The program is written in extended BASIC (Tektronix, 1976) for the 4051 desk-top computer with $32 \mathrm{~K}$ bytes of memory. The peripheral FP-51 ROM pack $^{1}$ is required for high-speed graphic and mathematical operations.

\section{Availability}

Listings for the game and analysis programs and additional information are available from the authors at no charge. Source code is also available on magnetic cartridge for a small fee. Make inquiry to Tektronix 
Applications Library, Group 451, Tektronix, Inc., P.0. Box 500, Beaverton, Oregon 97077.

\section{REFERENCE NOTE}

1. Kelly, R. T., Greitzer, F. L., \& Hershman, R. L. Air defense: A computer game for research in human performance (Tech. Rep.) San Diego: Navy Personnel Research and Development Center, in press.

\section{REFERENCE}

TeктRonix, Inc. 4051 graphic system reference manual. Beaverton, Ore: Author, 1976.

\section{NOTE}

1. MicroWorks, P.O. Box 1110, Del Mar, California 92014.

(Accepted for publication January 12, 1981.) 